\title{
Hierarchy Bloch Equations for the Reduced Statistical Density Operators in Canonical and Grand Canonical Ensembles
}

\author{
Liqiang Wei \\ Institute for Theoretical Atomic, Molecular and Optical Physics \\ Harvard University, Cambridge, MA 02318 \\ Chiachung Sun \\ Institute of Theoretical Chemistry, Jilin University \\ Changchun, Jilin 130023 P. R. China
}

November 1, 2018

\begin{abstract}
Starting from Bloch equation for a canonical ensemble, we deduce a set of hierarchy equations for the reduced statistical density operator for an identical many-body system with two-body interaction. They provide a law according to which the reduced density operator varies in temperature. By definition of the reduced density operator in Fock space for a grand canonical ensemble, we also obtain the analogous Bloch equation and the corresponding hierarchy reduced equations for the identical interacting many-body system. We discuss their possible solutions and applications.
\end{abstract}

$\underline{P A C S}$ 05.30.-d; 05.30.Fk; 05.30.Jp; 31.90.+s; 71.10.-w 


\section{Introduction}

The fundamental problem in quantum statistical mechanics is to determine the density matrix of a statistical ensemble. Once the density matrix is known, the thermodynamic properties can be calculated from the corresponding microscopic mechanical observables [1-4]. The basic equation of motion for the statistical density matrix is von Neumann-Liouville equation. For an equilibrium statistical ensemble, its solution takes the following special form,

$$
\rho=\sum_{i} \rho_{i}\left|\psi_{i}><\psi_{i}\right|
$$

where $\rho_{i}$ is the thermal probability distribution, and $\left|\psi_{i}\right\rangle$ is the energy eigenstate of the system. The summation over $i$ is for all the eigenstates consistent with symmetry constraints. For a canonical ensemble, for example, its relative thermal probability distribution and density operator are as follows,

$$
P_{i}=\exp \left(-\beta E_{i}\right)
$$

and

$$
D^{N}=\sum_{i} \exp \left(-\beta E_{i}\right)\left|\psi_{i}><\psi_{i}\right|=\exp \left(-\beta H_{N}\right),
$$

where $\beta$ is the reciprocal of the product of Boltzmann's constant $k_{b}$ and the absolute temperature $T$, and $H_{N}$ is the Hamiltonian of an $N$-body system. The trace of Eq. (3) gives the partition function of the canonical ensemble,

$$
\operatorname{Tr}\left(D^{N}\right)=\sum_{i} \exp \left(-\beta E_{i}\right)=Z(\beta, V, N)
$$

From their definition in Eqs. (1) or (4), we observe that, to determine the density matrix or the partition function, there are generally two steps 
involved. In the first step, we have to solve an energy eigenequation for the interacting many-body system to get its energy eigenstates and eigenvalues. In the second one, we need to sum over all the microscopic states. Obviously, these are both a very difficult or prohibitive task for the system considered, especially when the quantum effects are important and can not be neglected. Nevertheless, some other approaches or approximations have been developed, including the one called the equation of motion method, to address these issues $[5,6]$. Furthermore, for an identical interacting many-body system, it is known that its full density matrix conveys much more information than is necessary, and its reduced density matrix suffices to describe the states of a whole system. In analogous to that for the full density matrix, we attempt here to develop similar equations for the reduced density matrix so that they provide a kind of laws that the reduced density matrix obeys, or from which, the reduced density matrix can be directly determined.

This paper is organized as follows. In the next section, we derive the reduced Bloch equations for the reduced statistical density operators for a canonical ensemble. They are the equations of motion according to which the reduced density matrix varies in temperature. In Section 3, we define the reduced density matrix for a grand canonical ensemble, and obtain the analogous Bloch equation. We also extend the ideas developed in Section 2 on the reduced Bloch equation from the canonical ensemble to the case of a grand canonical ensemble. In Section 4, we summarize our results and discuss the possible routes for solving the equations as well as their applications. 


\section{Bloch Equation for the Reduced Statistical Density Operators in Canonical Ensemble}

Consider a canonical ensemble for an interacting $N$-body system whose density matrix is shown in Eq. (3). Differentiation of both sides of Eqs. (2) or (3) with respect to $\beta$ leads immediately to the differential equation,

$$
-\frac{\partial}{\partial \beta} D^{N}=H_{N} D^{N},
$$

which is called the Bloch equation $[5,6]$. It provides a law according to which the canonical density operator varies in temperature. It is equivalent to the definition (3). Nevertheless, it offers an alternative route to determine the density matrix. If the Hamiltonian of the system is known, we can directly solve the exact or approximate density matrix without recourse to the two procedures as we outline in the Introduction Section. A noticeable feature of Eq. (5) is that after its substitution of $\beta$ by $i t / \hbar$, it yields an equation that is identical in form with the time-dependent Schrödinger equation. Therefore, some developed techniques for solving the time-dependent Schrödinger equation can be borrowed to obtain the solutions for the Bloch equation (5).

In practical applications, moreover, the interactions among the identical particles such as electrons in atoms, molecules, and solids are often twobody forces. The $N$ th-order density matrix contains much more information than is required. In order to calculate the thermodynamic properties of the system, we need at most the second-order reduced density matrix and not the full $N$ th-order one. That is to say, the $p$ th-order reduced density matrix can describe the $N(>p \geq 2)$ particle states. For this purpose, we try to find a method for directly solving the $p$ th-order reduced density matrix instead 
of the one for a full $N$ th-order density matrix.

The $p$ th-order reduced density matrix of an $N$-particle system is generally defined by [7-11]

$$
D^{p}=L_{N}^{p}\left(D^{N}\right)
$$

where $L_{N}^{p}$ is the contraction operator $[12,13]$. Its trace gives the partition function defined before

$$
\operatorname{Tr}\left(D^{p}\right)=Z(\beta, V, N)
$$

Assume that the Hamiltonian of the system considered is made up of two contributions,

$$
H_{N}=\sum_{i=1}^{N} h(i)+\sum_{i<j}^{N} g(i, j)
$$

where $h(i)$ and $g(i, j)$ are one- and two-particle operators, respectively. Rewrite the $H_{N}$ into

$$
H_{N}=H_{1}^{p}+\sum_{j=p+1}^{N} h(i)+\sum_{i=1}^{p} \sum_{j=p+1}^{N} g(i, j)+\sum_{i<j(i \geq p+1)}^{N} g(i, j),
$$

where

$$
H_{1}^{p}=\sum_{i=1}^{p} h(i)+\sum_{i<j}^{p} g(i, j)
$$

Then substituting the Hamiltonian Eq. (9) into the Bloch equation (5) and applying on both sides the operator $L_{N}^{p}$, we get the following equations for the reduced density operators,

$$
\begin{aligned}
-\frac{\partial}{\partial \beta} D^{p}= & H_{1}^{p} D^{p}+(N-p) L_{p+1}^{p}\left[h(p+1) D^{p+1}\right]+(N-p) L_{p+1}^{p}\left[\sum_{i=1}^{p} g(i, p+1) D^{p+1}\right]+ \\
& +\left(\begin{array}{c}
N-p \\
2
\end{array}\right) L_{p+2}^{p}\left[g(p+1, p+2) D^{p+2}\right] .
\end{aligned}
$$


We call them the reduced Bloch equations for the canonical ensemble. They relates the $D^{p}, D^{p+1}$, and $D^{p+2}$ in a certain way, and can be seen as a set of hierarchy. From the general definition for the reduced density matrix (6), they can also be regarded as an independent equation for $D^{p+2}$. Similar procedures have lead to the hierarchy equations for the reduced density matrix of the pure states before from the Schrödinger equation in a density matrix form [14-17]. It follows from the idea in deriving the Bogoliubov-Born-Green-Yvon (BBGKY) hierarchy in classical statistical mechanics [4].

\section{Bloch Equation for the Reduced Statisti- cal Density Operators in Grand Canonical Ensemble}

To provide more avenues for studying the behaviors of statistical density matrices, the above description can also be extended to the case of a grand canonical ensemble. In $N$-particle Hilbert space $V^{N}$, the relative grand canonical distribution reads

$$
P_{i}(N)=\exp \left[-\beta\left(E_{i}-\mu N\right)\right]
$$

and the density matrix is

$$
\begin{aligned}
D_{G}(N) & =\exp [-\beta(H-\mu N)] \\
& =\exp (-\beta \bar{H})
\end{aligned}
$$

where

$$
\bar{H}=H-\mu N
$$


is called the grand Hamiltonian on $V^{N}$. Again, upon differentiating both sides of Eq. (13) with respect to $\beta$, we have

$$
-\frac{\partial}{\partial \beta} D_{G}(N)=\bar{H} D_{G}(N)
$$

which has the same form as Eq. (5). We call it the Bloch equation for the grand canonical ensemble on $N$-particle Hilbert space $V^{N}$.

For the grand canonical ensemble, the number of particles $N$ fluctuates. Its density matrix should be defined in the entire Fock space: $F=\sum_{N} \oplus V^{N}$. That is, it should be written as the direct sum of every density matrix $D_{G}(N)$ belonging to the $N$-particle space $V^{N}$,

$$
D_{G}=\sum_{N=0}^{\infty} \oplus D_{G}(N)
$$

and its trace in Fock space is the grand partition function,

$$
\operatorname{Tr}\left(D_{G}\right)=\sum_{N=0}^{\infty} \sum_{i} \exp \left[-\beta\left(E_{i}-\mu N\right)\right]=\Xi(\beta, \mu, V) .
$$

For the same consideration, we define the corresponding $p$ th-order reduced density matrix as follows,

$$
D_{G}^{p}=\sum_{N=p}^{\infty} \oplus\left(\begin{array}{c}
N \\
p
\end{array}\right) L_{N}^{p}\left[D_{G}(N)\right]
$$

where the binomial coefficient $\left(\begin{array}{c}N \\ p\end{array}\right)$ is added to reflect the symmetric property of the density matrix in terms of the permutation of particles. Obviously,

$$
D_{G}^{0}=\Xi(\beta, \mu, V),
$$

and

$$
\operatorname{Tr}\left(D_{G}^{p}\right)=\left\langle\left(\begin{array}{c}
N \\
p
\end{array}\right)\right\rangle D_{G}^{0}
$$


Assume Hamiltonian $H$ in $\bar{H}$ takes the same form as in Eq. (8). Rewrite $\bar{H}$ into

$$
\bar{H}=\bar{H}_{1}^{p}+\sum_{j=p+1}^{N} \bar{h}(i)+\sum_{i=1}^{p} \sum_{j=p+1}^{N} g(i, j)+\sum_{i<j(i \geq p+1)}^{N} g(i, j),
$$

where

$$
\bar{h}(i)=h(i)-\mu,
$$

and

$$
\bar{H}_{1}^{p}=\sum_{i=1}^{p} \bar{h}(i)+\sum_{i<j}^{p} g(i, j) .
$$

Substituting the Eq. (21) into Eq. (15) and applying on both sides the operator $\sum_{N=p}^{\infty} \oplus\left(\begin{array}{c}N \\ p\end{array}\right) L_{N}^{p}$, we finally obtain the equations for the reduced density matrix

$$
\begin{aligned}
-\frac{\partial}{\partial \beta} D_{G}^{p}= & \bar{H}_{1}^{p} D_{G}^{p}+(p+1) L_{p+1}^{p}\left[\bar{h}(p+1) D_{G}^{p+1}\right]+(p+1) L_{p+1}^{p}\left[\sum_{i=1}^{p} g(i, p+1) D_{G}^{p+1}\right]+ \\
& +\left(\begin{array}{c}
p+2 \\
2
\end{array}\right) L_{p+2}^{p}\left[g(p+1, p+2) D_{G}^{p+2}\right],
\end{aligned}
$$

which are the reduced Bloch equation on Fock space for the grand canonical ensemble. They also represents a law accoding to which the grand reduced density matrix changes in temperature. Similar notes can also be made as in the case for the canonical ensemble.

\section{Summary and Discussions}

In this paper, within the framework of equilibrium quantum statistical mechanics, we have derived the hierarchy equations of the density operators for both canonical and grand canonical ensembles. They are the equations 
of motion in terms of the variation of temperature that the reduced density operator satisfies. They provide the route for a direct determination of the reduced density operator for the statistical ensembles.

In the first place, the Eqs. (11) or (24) can be solved in an approximate way. Since they are a set of hierarchy equations, we have to relate the $D^{p+1}$ and the $D^{p}$ in a certain way to decouple them. The most obvious choice is to take an orbital approximation. For an identical fermion system, this means to express a $p$ th-order reduced density matrix as a Grassmann product of $p$ first-order reduced density matrices as follows

$$
\begin{aligned}
D^{p+1} & =D^{p} \wedge D^{1} / D^{0} \\
& =\underbrace{D^{1} \wedge D^{1} \wedge \ldots \wedge D^{1}}_{p+1} /\left(D^{0}\right)^{p} .
\end{aligned}
$$

Similar scheme has been applied for solving the reduced Schrödinger equation for the reduced density matrix to obtain the usual Hartree-Fock equation [14-17]. We expect that it will lead to more general equation beyond that for zero temperature when it is applied to the Eq. (24) [21]. Consequently, this will provide a possibility for studying the microscopic structure of high temperature species or macroscopic molecules at different temperatures [19-22]. In addition, it will serve as a staring point to develop more general schemes for studying such important issues as the interplay between the microscopic structure and macroscopic thermodynamic properties [18]. This also includes the pressure effects on the electronic structure for solids [23]. Furthermore, Eqs. (11) or (24) can also be solved exactly for some model systems. One example is to consider a pairing Hamiltonian, and solve the equations in an occupation number representation [25-27]. The pairing Hamiltonian is 
a useful model, for example, in the study of the nuclear structure and the microscopic states of superconductors. We hope that we can obtain its exact reduced density matrices with different orders and therefore investigate the corresponding thermodynamic properties [28]. Work along these two lines can be carried out.

\section{References}

[1] R. C. Tolman, The Principles of Statistical Mechanics (Oxford University Press, 1938).

[2] R. P. Feynman, Statistical Mechanics (W. A. Benjamine, Reading, Mass., 1972).

[3] D. Chandler, Introduction to Modern Statistical Mechanics (Oxford University, 1987).

[4] K. Huang, Statistical Mechanics (John Wiley \& Sons, 2nd Edition, 1987).

[5] F. Bloch, Zeits. f. Physick 74, 295 (1932).

[6] J. G. Kirkwood, Phys. Rev. 44, 31 (1933).

[7] K. Husimi, Proc. Phys. Math. Soc. Japan, Ser. 3 32, 264 (1940).

[8] P. O. Löwdin, Phys. Rev. 97, 1474 (1955).

[9] U. Fano, Rev. Mod. Phys. 29, 74 (1957). 
[10] R. McWeeny, Rev. Mod. Phys. 32, 335 (1960).

[11] E. R. Davidson, Reduced Density Matrices in Quantum Chemistry (New York: Academic Press, 1976).

[12] K. Kummer, J. Math. Phys. 8, 2063 (1967).

[13] J. E. Harriman, Phys. Rev. A 17, 1257 (1978).

[14] L. Cohen and C. Frishberg, Phys. Rev. A 13, 927 (1976).

[15] H. Nakatsuji, Phys. Rev. A 14, 41 (1976).

[16] H. Schlosser, Phys. Rev. A 15, 1349 (1977).

[17] J. E. Harriman, Phys. Rev. A 19, 1893 (1979).

[18] P. O. Löwdin, Intern. J. Quantum Chem. Vol xxix, 1651 (1986).

[19] W. Klemperer, Ann. Rev. Phys. Chem. 46, 1 (1995).

[20] K. Pichler, D. A. Halliday, D. D. C. Bradley, P. L. Burn, R. H. Friend, and A. B. Holmes, J. Phys.: Condens. Matter 5, 7155 (1993)

[21] J. Yu, M. Hayashi, S. H. Lin, K.-K. Liang, J. H. Hsu, W. S. Fann, C.-I. Chao, K.-R. Chuang, S.-A. Chen, Synth. Met. 82, 159 (1996).

[22] S. P. Kwasniewski, J. P. Francois, and M. S. Deleuze, J. Phys. Chem A 107,5168 (2003).

[23] H. G. Drickamer and C. W. Franck, Electronic Transitions and the High Pressure Chemistry and Physics of Solids (London, Chapman and Hall, New York, 1973). 
[24] L. Wei and C. C. Sun, submitted (2003); cond-mat/0306307.

[25] L. S. Kisslinger and R. A. Sorensen, Mat. Fys. Medd. Dan. Vid. Selsk. 32, no 12 (1960).

[26] S. G. Nilsson and O. Prior, Mat. Fys. Medd. Dan. Vid. Selsk. 32, no 16 (1960).

[27] A. J. Coleman, J. Math. Phys. 6, 1425 (1965).

[28] L. Wei and C. C. Sun (2003), in preparation. 